\title{
La gouvernance de nos établissements est-elle adlaptée au nouveau financement hospitalier?
}

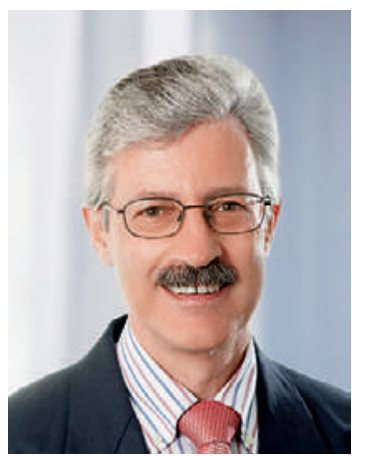

Cela fait 18 mois que le nouveau financement hospitalier est en vigueur dans notre pays. Les patients sont toujours traités de manière adéquate et restent majoritairement satisfaits. La possibilité d'aller se faire traiter dans un autre canton n'a pas engendré de mouvement particulier. Pour les médecins hospitaliers, la charge administrative reste considérable mais la liberté thérapeutique n'est pas menacée. Cette stabilité est remarquable dans le contexte d'une structure tarifaire évolutive: en effet, nous vivons déjà la $2^{\mathrm{e}}$ version de SwissDRG et la $3^{\mathrm{e}}$ vient d'être présentée. Les différences d'une année à l'autre sont parfois importantes (cf. l'article de B. Meyer et P. Ingenpass à la page 1018), témoignant du dynamisme de notre système.

Ces aspects positifs ne doivent néanmoins pas occulter les difficultés rencontrées dans nos hôpitaux. Hormis le fait que des taux de base différenciés sont encore appliqués pour compenser l'absence de finesse de la structure tarifaire, de grandes incertitudes subsistent, rendant la planification des budgets hospitaliers complexe. Les fortes variations des poids relatifs de coût d'une version à l'autre, de même que la modification des durées de séjour de certains groupes, sont difficilement prévisibles. Il en résulte des négociations parfois chaotiques avec les assureurs, aboutissant régulièrement à un prix de base inférieur à l'année précédente. L'intransigeance de M. Prix faisant fi de l'esprit de la révision de la LAMal n'est pas étrangère à cela. Enfin, les cantons constatent une relative fluctuation de leurs recettes: la générosité de l'Etat n'est donc plus de mise, les prestations d'intérêt général étant les premières à subir des coupes parfois draconiennes.

\section{La concurrence entre établissements publics et privés s'exacerbe.}

Conjuguée à la pénurie de médecins et de personnel soignant qualifié, la concurrence entre établissements publics et privés s'exacerbe. Les grands hôpitaux n'arrivent plus à répondre à la demande de leur région, favorisant l'éclosion de cliniques spécialisées dans des domaines particuliers où la demande est forte. Certains établissements sont encore sous la tutelle d'un contrôle étatique tatillon, contraints d'effectuer leurs procédures d'adjudication selon les règles du marché public et limités par des contrats de prestations très restrictifs. Confrontés à une baisse de leurs revenus aussi bien de la part des assureurs que de l'Etat, ils n'ont pas la liberté de développer rapidement des activités compensatoires. Représentant souvent un des plus gros employeurs locaux, ils ne peuvent procéder à aucune baisse de salaire ni licenciement.

Il est donc indispensable que nos hôpitaux puissent bénéficier d'une gouvernance réactive. Contrôlée par des indicateurs crédibles, la qualité doit être produite, documentée, validée puis communiquée. Des processus modernes doivent pouvoir être implémentés, tels qu'ERAS (Enhanced Recovery

\section{Il est indispensable que nos hôpitaux puissent bénéficier d'une gouvernance réactive.}

After Surgery) qui permet de simplifier les soins, d'éviter les complications, d'améliorer les résultats, de diminuer la durée de séjour et de faire baisser les coûts de manière significative. Qualité et économicité ne sont donc pas antagonistes mais plutôt synergiques.

Face à la pression financière et d'image qui s'installe, nos établissements hospitaliers doivent être créatifs. Pour cela, il est nécessaire qu'ils puissent jouir de la liberté requise. Pour être efficace, leur direction doit posséder une forte composante médicale. Une étude britannique à large échelle, à paraître prochainement, prouve en effet une meilleure satisfaction des patients et une meilleure qualité des résultats dans les établissements dirigés par des médecins. Pour être performants, nos hôpitaux ont ainsi besoin d'une gouvernance éclairée dont l'entente entre administrateurs, soignants et médecins est garante de la qualité des prestations, du bienêtre des patients, de l'ambiance générale de travail et finalement de la bonne santé économique du système de santé régional.
Dr Pierre-François Cuénoud, vice-président de la FMH, responsable du domaine Tarifs et économie de la santé pour les médecins hospitaliers 